\title{
Hypothalamic Structural and Functional Imbalances in Anorexia Nervosa
}

\author{
Vincent Florent ${ }^{a-c}$ Marc Baroncini ${ }^{a, b, d, m}$ Patrice Jissendi-Tchofo ${ }^{e, f}$ \\ Renaud Lopes ${ }^{\mathrm{e}}$ Matthieu Vanhoutte ${ }^{\mathrm{e}}$ Sowmyalakshmi Rasika ${ }^{9}$ \\ Jean-Pierre Pruvo $^{b, e}$ Jean Vignau ${ }^{h}$ Stephane Verdun ${ }^{i}$ Jeanette E. Johansen ${ }^{j}$ \\ Marie Pigeyre $^{b, k, l}$ Sebastien G. Bouret ${ }^{a, b, m}$ Ida A.K. Nilsson ${ }^{j}$ Vincent Prevot ${ }^{a, b}$ \\ a Inserm, Laboratory of Development and Plasticity of the Neuroendocrine Brain, Lille, France; bSchool of Medicine, \\ Université Lille, Lille, France; ${ }^{c}$ Nutrition, Arras General Hospital, Lille, France; ${ }^{d}$ Neurosurgery, CHU Lille, Lille, France; \\ eNeuroradiology, CHU Lille, Lille, France; ${ }^{f} \mathrm{CHU}$ Saint-Pierre, ULB, Brussels, Belgium; ${ }^{9}$ APHP-R DEBRE, Protect, \\ University Hospital, Paris, France; ${ }^{\text {h}}$ Psychiatry, CHU Lille, Lille, France; ' ${ }^{B}$ Biostatistics Department of the GHICL, \\ Lomme, France; jDepartment of Molecular Medicine and Surgery and Center for Molecular Medicine, Karolinska \\ Institutet/Hospital, Stockholm, Sweden; 'Nutrition, CHU Lille, Lille, France; 'U1190, European Genomic Institute for \\ Diabetes, Lille, France; ${ }^{m}$ The Saban Research Institute, Developmental Neuroscience Program and Diabetes and \\ Obesity Program, Center for Endocrinology, Diabetes and Metabolism, Children's Hospital Los Angeles, University \\ of Southern California, Los Angeles, CA, USA
}

\section{Keywords}

Magnetic resonance imaging $\cdot$ Tractography $\cdot$ Spectroscopy $\cdot$ Energy homeostasis · Appetite $\cdot$ Neurodevelopmental disorder $\cdot$ Human beings

\begin{abstract}
The hypothalamus contains integrative systems that support life, including physiological processes such as food intake, energy expenditure, and reproduction. Here, we show that anorexia nervosa (AN) patients, contrary to normal weight and constitutionally lean individuals, respond with a paradoxical reduction in hypothalamic levels of glutamate/ glutamine (Glx) upon feeding. This reversal of the Glx response is associated with decreased wiring in the arcuate nucleus and increased connectivity in the lateral hypothalamic area, which are involved in the regulation on a variety of physiological and behavioral functions including the con-
\end{abstract}

trol of food intake and energy balance. The identification of distinct hypothalamic neurochemical dysfunctions and associated structural variations in AN paves the way for the development of new diagnostic and treatment strategies in conditions associated with abnormal body mass index and a maladaptive response to negative energy balance.

๑) 2019 S. Karger AG, Basel

\section{Introduction}

Anorexia nervosa (AN) is a severe and complex mental disorder mainly affecting young adult women $[1,2]$. Patients with AN present with low body weight in conjunc-

V.F. and M.B. contributed equally to this work. M.B., I.A.K.N., and V.P. jointly directed this work.
Vincent Prevot, $\mathrm{PhD}$

Inserm 1172, Bâtiment Biserte Place de Verdun

FR-59045 Lille Cedex (France)

E-Mail vincent.prevot@inserm.fr 
tion with self-induced starvation, intense fear of gaining weight, and body image distortion [3]. One of the most questioning features of individuals with AN is their ability to chronically restrict energy intake, whereas they are in an emaciated condition often for many years, and not rarely to the point of death. These alterations of interoception clearly illustrate a communication breakdown between the body and the brain, that is, the inability of the brain to translate circulating factors that signal the energy state of the organism into the appropriate adaptive behavior [4]. In fact, recent genome-wide association studies provide evidence of a genetic predisposition for a lower body weight set-point in AN and also indicate that it is not merely a psychiatric but also a metabolic disorder [5, 6]. However, among the wealth of functional magnetic resonance imaging (MRI) studies on the brain of AN patients [7], few if any have examined differences in the hypothalamus, a region crucially involved in perceiving and integrating metabolic signals. Moreover, very little is known about the mechanisms by which the brain reacts to acute energetic challenges such as those caused by the ingestion of a meal, either physiologically or in eating disorders. Deciphering these mechanisms is thus a prerequisite not only to obtaining insight into appropriate food intake regulation in healthy individuals but also into how its deregulation could subtend pathological conditions such as AN.

To explore differences in brain structure and function in patients with AN and control subjects, and thus gain insight into the putative neurobiological mechanisms underlying maladaptive food intake, we used proton magnetic resonance spectroscopy (MRS) [8] to measure brain metabolites as an index of function, and a probabilistic tractography algorithm [9] combined with diffusion-tensor imaging [10] to measure connectivity in the hypothalamus. Hunger and the hunger-mediated perception of food as a reward are thought to be mediated by circuits linking energy state to motivational drive to eat, which involves distributed and interconnected neuronal networks [11-14]. One of these networks includes the arcuate nucleus of the hypothalamus (ARH) [12] that communicates with extra-hypothalamic structures such as the thalamus, which in turn project to cortical areas that integrate sensory cues and the energy needs of the body [15]. Appetite, the reward value of feeding and motor actions associated with ingestive behaviors also involve the lateral hypothalamic area (LHA) $[13,16,17]$, which is bidirectionally connected with $>50$ distinct brain areas including in the midbrain, brainstem, and the telencephalon $[17,18]$.

Hypothalamic Defects in AN

\section{Materials and Methods}

\section{Subjects}

The study was carried out with the approval of the Comite de Protection des Personnes Nord Ouest IV (No. EudraCT: 2012A01331-42), and informed consent was obtained from all included subjects. Three groups of 10 female subjects with a mean age of $23.5 \pm 0.8$ were included (because only $10 \%$ of AN patients are males); all participants were under oral contraception to avoid any cofounding effects of the menstrual cycle on the hypothalamus [8], except 6 out of 10 women with AN who were in hypothalamic amenorrhea (i.e., an arrest of the menstrual cycle). Importantly, circulating estradiol levels in women receiving oral contraceptives, usually in the range of $20-30 \mathrm{ng} / \mathrm{L}$ [19], is comparable to the one found in AN patients with hypothalamic amenorrhea [20] and both type of subjects show low or absent pulsatile secretion of luteinizing hormone $[21,22]$.

Patients included in the study were diagnosed with AN of a restrictive subtypeaccording to the Diagnostic and Statistical Manual of Mental Disorders, 4 th Edition, at least 1 year prior to the study $(n=10, \mathrm{BMI}<18)$, and were in treatment at the eating disorders unit of Lille university hospital. From a prescreening of 185 volunteers corresponding to the inclusion criteria, patients were selected according to a correspondence between the 13th or 14th day of the pill-induced menstrual cycle (the phase of the artificial menstrual cycle in which the hypothalamus is fully inhibited with regard to reproductive function, most similar to hypothalamic amenorrhea) and available time-slots for MRI for clinical research. Two control groups were constituted from among selected volunteers: normalweight controls $(n=10,20<\mathrm{BMI}<25)$ and individuals with constitutional leanness $(n=10, \mathrm{BMI}<18.5)$, defined as nonpathological underweight that does not meet the Diagnostic and Statistical Manual of Mental Disorders, 4th Edition criteria for AN of a restrictive. No subjects included in any of the 2 control groups had a personal or family history of eating disorders or a food cognitive restraint score lower than 13 on the 3 -factor eating questionnaire [23]. The predicted basal energy expenditure of each volunteer was calculated using the Harris and Benedict equation [24].

The incidence of treatment-seeking AN patients being very low, that is, about 8 per 100,000 person-years [1], the recruitment of the 10 AN patients willing to participate to the study required $>1$ year. This sample size was chosen in order to give sufficient power to detect a difference in metabolic MRI activity in the same subject before and after food intake as well as in connectivity between groups. This number compares favorably with pervious MRS $[8,25]$ and diffusion weighed imaging studies $[8,26]$ that have been conducted in the human hypothalamus. This, together with the aforementioned stringent screening of the population of subjects to restrict as much as possible inter-individual variability, ensured adequate power to detect significant differences while also allowing for natural variation in responses (online suppl. Table S1-S5; for all online suppl. material, see www.karger.com/doi/10.1159/000503147).

\section{Investigative Protocol}

The participants had a first MRI after overnight fasting at 08:00 a.m. This first MRI session lasted $45 \mathrm{~min}$ and comprised 1 morphological sequence and 3 functional sequences. The participants were then asked to ingest a nutritional supplement as breakfast. This breakfast was calibrated to $25 \%$ of the predicted basal energy expenditure for each participant (Table 1). One hour after 
Table 1. Characteristics of the participants according group

\begin{tabular}{|c|c|c|c|c|c|c|}
\hline & $\begin{array}{l}\text { Control } \\
\text { group } \\
(n=10)\end{array}$ & $\begin{array}{l}\text { Constitutional } \\
\text { thinness group } \\
(n=10)\end{array}$ & $\begin{array}{l}\text { Anorexia } \\
\text { nervosa group } \\
(n=10)\end{array}$ & $\begin{array}{l}p \text { value } \\
\text { CTL vs. CL }\end{array}$ & $\begin{array}{l}p \text { value } \\
\text { CTL vs. AN }\end{array}$ & $\begin{array}{l}p \text { value } \\
\text { CL vs. AN }\end{array}$ \\
\hline Weight, kg & $61.8 \pm 2.1$ & $46.4 \pm 0.9$ & $42.6 \pm 1.8$ & $<0.0001$ & $<0.0001$ & 0.2761 \\
\hline BMI, $\mathrm{kg} / \mathrm{m}^{2}$ & $21.9 \pm 0.4$ & $17.1 \pm 0.3$ & $15.3 \pm 0.6$ & $<0.0001$ & $<0.0001$ & 0.0244 \\
\hline \multicolumn{7}{|l|}{ Administered oral nutritional } \\
\hline supplement $2 \mathrm{kcal} / \mathrm{mL}$ (volume in $\mathrm{mL}$ ) & $264 \pm 6.8$ & $240 \pm 8.5$ & $216 \pm 6.8$ & 0.0675 & 0.0003 & 0.0853 \\
\hline Calorie intake, kcal & $529 \pm 14$ & $480 \pm 17$ & $433 \pm 14$ & 0.0706 & 0.0003 & 0.0873 \\
\hline
\end{tabular}

Data are expressed as mean \pm SEM.

CTL versus CL significance of the statistical difference between the control group (CTL) and the constitutional lean group (CL). CTL versus AN significance of the statistical difference between the control group (CTL) and the anorexia nervosa group (AN). CL versus AN significance of the statistical difference between the constitutional lean group (CL) and the anorexia nervosa group (AN).

$\mathrm{AN}$, anorexia nervosa; BMI, body mass index; TFEQ, three factor eating questionnaire; BEE, basal energy expenditure.

food intake, the participants were subjected to a second 45 -min MRI session (i.e., at 10:00 a.m.). Importantly, all subjects, including AN patients, had volunteered to ingest this calibrated breakfast, which was consumed together with a medical team trained for the purpose, in order to limit the anxiety of patients.

\section{Image Acquisition}

All MRI exams were performed at the University Hospital of Lille on a 3T Philips Achieva scanner (Philips Healthcare, Best, the Netherlands) using an 8-channel phased-array head coil and a whole-body coil transmission. First, high-resolution three-dimensional T1-weighted images were acquired as a series of thick 176 sagittal slices, using a Turbo Field Echo sequence $(\mathrm{TR}=7.20 \mathrm{~ms}$; $\mathrm{TE}=3.3 \mathrm{~ms}$; flip-angle $=9^{\circ}$; voxel size $=1 \times 1 \times 1 \mathrm{~mm}^{3}$, FOV $=$ $256 \times 256 \times 176 \mathrm{~mm}^{3}$ ). Then, 2 diffusion-weighted imaging were performed using a single-shot Echo-Planar Imaging sequence in normal and reversed phase-encoding polarity, for further distortion correction $\left(\mathrm{TR}=13 \mathrm{~s}, \mathrm{TE}=55 \mathrm{~ms}\right.$, flip angle $=90^{\circ}$; voxel size $=$ $2 \times 2 \times 2 \mathrm{~mm}^{3}, \mathrm{FOV}=256 \times 256 \mathrm{~mm}^{2}, 66$ slices, acceleration factor of 2, 64 gradient-encoded directions with a b-value of $1,000 \mathrm{~s} / \mathrm{mm}^{2}$, one $b=0 \mathrm{~s} / \mathrm{mm}^{2}$ image). Finally, 2 PRESS localized spectra were acquired over hypothalamus and thalamus volumes $(\mathrm{TR}=2,000$ $\mathrm{ms}, \mathrm{TE}=37 \mathrm{~ms}, \mathrm{FOV}=10 \times 10 \times 10 \mathrm{~mm}^{3}$ and a spectral resolution of $1.95 \mathrm{~Hz} /$ point). In the hypothalamus, the voxel used for spectroscopy was placed between the plate numbers 31 and 41 of the atlas of Mai et al. [27] on the wall of the third ventricle and just above the optic tract. In the thalamus, the voxel was placed between the plates numbers 45 and 52 [27], 5 and $15 \mathrm{~mm}$ above the bi-commissural line, and $5 \mathrm{~mm}$ lateral from the midline. While the voxel did not include the paraventricular thalamic nucleus, which lies at the immediate proximity of the third ventricle, it comprised mainly the mediodorsal thalamic nucleus and the posterior ventrolateral nucleus, and some of the lateral and medial thalamic ventroposterior nuclei as well as the centromedial thalamic nucleus at the most posterior aspect of the voxel.

\section{Spectral Analysis}

The absolute metabolite concentrations were obtained using the LCmodel [28] quantification algorithm. Within LCmodel, concentrations of metabolites are determined using a linear combination fit of the individual in vitro metabolite spectra that comprise the "basis set" to the in vivo data. The basis set was acquired with long TR and short TE to minimize relaxation effects. Only voxels within the excitation region were presented for LCmodel analysis without any other preprocessing such as zero filling or apodization. Peak registration in LCmodel was performed using the prominent peaks of glutamine, glutamate, and creatine observed inside hypothalamus volume of interest, while using thalamus as control volume of interest. In the spectral analysis window, 3.85-1.0 ppm was used for 3T data to prevent variability in the results from lipid artifacts that are more prominent at $3 \mathrm{~T}$. Spectra were included in the final analysis based on quality criteria defined by objective output parameters from the LCmodel analysis: sufficient spectral resolution (full width at half minimum $0.07 \mathrm{ppm}$ ), sufficient information (SNR 4), and residuals that were randomly scattered about zero to indicate a reasonable fit to data. By using the analysis window of $3.85 \mathrm{ppm}$, deviations in the fit due to baseline distortion from improper water suppression were avoided. All spectra included in the results had smooth and reasonably flat baseline estimates.

\section{Processing of T1-Weighted Images}

T1-weighted images were processed with the Freesurfer software package (version 5.3, http://surfer.nmr.mgh.harvard.edu/), which provided nonuniformity and intensity correction, skull stripping, and gray/white matter segmentation (box 1 in online suppl. Fig. S4). The 34 regions of interest (ROIs), constituting the sub-segmentation of the hypothalamus, were manually defined on each T1-weighted patient image (box 2 in online suppl. Fig. S4) based on anatomy (online suppl. Fig. S2) and using the MRI atlas of the human hypothalamus [10], as a reference. T1-weighted im- 
ages were coregistered onto b0 images using a rigid-body model implemented in the Statistical Parametric Mapping software version 12 (http://www.fil.ion.ucl.ac.uk/spm/; box 3 in online suppl. Fig. S4). Coregistration between T1-weighed and b0 images was validated by a neuroradiologist expert from visual assessment. All hypothalamic ROIs defined in $\mathrm{T} 1$ space had to be transformed into diffusion space, where all calculations were done, using the coregistration matrix above. The white matter mask was computed by floodfilling the interior of the white surface in intersection with the brain mask, creating a volume containing both white matter and ROIs defining subsegmentation of the hypothalamus.

\section{Processing of Diffusion-Weighted Imaging}

First, each diffusion-weighted imaging (DWI) dataset was aligned to its b0 image using affine registration to correct for patient motion and Eddy currents using FSL (FMRIB Software Library, http://www.fmrib.ox.ac.uk/fsl/; box a in online suppl. Fig. S4). Then, CMTK (Computational Morphometry Toolkit, https:// www.nitrc.org/projects/cmtk/) was used to estimate the deformation field from opposite phase-encoding polarity (box b in online suppl. Fig. S4) and apply correction to DWI images (box c in online suppl. Fig. S4) to get free images from geometric distortions [29]. Diffusion tensors were fitted at each voxel (box d in online suppl. Fig. S4) to calculate fractional anisotropy (FA) and mean diffusivity maps (box e in online suppl. Fig. S4). Whole-brain tractography was done with the MRtrix software package version 0.2.12 (Brain Research Institute, Melbourne, Australia, http://www.brain.org. au/software/; boxes $\mathrm{f}^{-\mathrm{f}}$ "' in online suppl. Fig. S4). Fiber orientation distribution (FOD) at highly anisotropic voxels $(\mathrm{FA}>0.7)$ determined the response function (box $\mathrm{f}$ in online suppl. Fig. S4), which was then used for constrained spherical deconvolution (box $\mathrm{f}^{\prime}$ in online suppl. Fig. S4) to accurately estimate the FOD [30]. Fibers were then generated with a probabilistic tracking algorithm that samples FOD at each step [9]. The algorithm generated 150,000 fibers of minimum length $20 \mathrm{~mm}$. All voxels in the $1-\mathrm{mm}$ dilated white matter mask were used as seeds, and the tracking procedure was stopped if a fiber reached a voxel outside the mask or if a stop criterion was met (high fiber curvature or low FOD; box $\mathrm{f}^{\prime \prime}$ " in online suppl. Fig. S4). Finally, 2 features were computed for each coregistered ROI defining subsegmentation of the hypothalamus: (i) connectivity information is computed from whole-brain tractography as the number of fibers passing through the ROI (box $\mathrm{g}$ in online suppl. Fig. S4) and (ii) the mean diffusivity/FA inside each of these ROI (box e in online suppl. Fig. S4). Importantly, there was no difference between participants in the ratio of the volume of the distinct ROIs under scrutiny to the intracranial volume. A visual quality control of the tractography was made based on well-known bundles as the postcommissural fornix and the principal mammillary bundle passing through the hypothalamus. These bundles were used as reference to exclude any aberrant tractography.

\section{Statistics}

All analyses were performed using Prism 7 (GraphPad Software) and assessed for normality (D'Agostino and Pearson normality test) and variance, when appropriate. Even though the study was conducted with 10 subjects in each group, the number of independent values plotted in the graphs $(n)$ varied between 8 and 10, because 1 of the 2 spectra acquired during functional MRS was not exploitable or DWI data were lacking for a given ROI. Data had normal distributions and were compared using a one-way ANOVA for multiple comparisons or a two-way repeated measures ANOVA. The significance level was set at $p<0.05$. Data are indicated as means \pm SEM. The number of biologically independent experiments, $p$ values, and degrees of freedom are indicated either in the main text or in the figure legends.

\section{Results and Discussion}

\section{The Hypothalamic Glutamatergic Response to Food}

Intake Is Markedly Altered in AN

In order to determine whether hypothalamic neuronal activity responded differently to food intake in patients with AN as compared to control women, we used taskrelated proton MRS, whose sensitivity makes it suitable for the study of functional changes [31]. We compared brain concentrations of glutamine/glutamate $(\mathrm{Glx})$ as a marker of glutamatergic (dys)function, between women newly hospitalized for AN and healthy control women both before and after feeding (Table 1); other metabolites - N-acetyl aspartate (NAA), myoinositol (Ins), and choline (Chol) and creatin (online suppl. Fig. S1) were used to estimate overall neural integrity and function. A third group of women displaying constitutional leanness, that is, a body mass index comparable to that of AN patients but with no food dysphoria, were also investigated (Table 1) [32]. Participants underwent an overnight fast, followed by 2 multimodal MRI sessions before and after the ingestion of a calibrated breakfast, respectively. Voxel-based spectroscopic analyses of freely moving molecules were performed both in the hypothalamus and in the thalamus, and data were normalized to creatine, in a relative quantitative approach (Fig. 1, online suppl. Fig. S1). In the hypothalamus, the feeding-evoked response of the Glx-to-creatine ratio differed significantly between the groups (one-way ANOVA: $F_{(2,23)}=5.807, p=0.009$, $n=8-10$ women per group. Tukey's multiple comparison test: control vs. AN, $q_{(23)}=4.42, p=0.013$; constitutional leanness vs. AN, $q_{(23)}=3.97, p=0.026$; control vs. constitutional leanness, $q_{(23)}=0.69, p=0.877$, online suppl. Table S1; Fig. 1b). The ratio also varied at baseline, that is, under fasting conditions, between the 3 groups (Fig. 1c). Thus, while the mean Glx-to-creatine ratio was significantly higher before ingestion of the calibrated breakfast in women with AN than in healthy women (two-way repeated measure ANOVA: interaction, $F_{(2,23)}=5.807, p=$ 0.009 ; subjects, $F_{(2,23)}=0.656, p=0.528$; time, $F_{(1,23)}=$ $0.181, p=0.674$; Sydac's multiple comparison test: Control fasting versus AN fasting $t_{(46)}=2.767, p=0.020, n=$ 8 persons per group, online suppl. Table S2; Fig. 1c), $1 \mathrm{~h}$ 

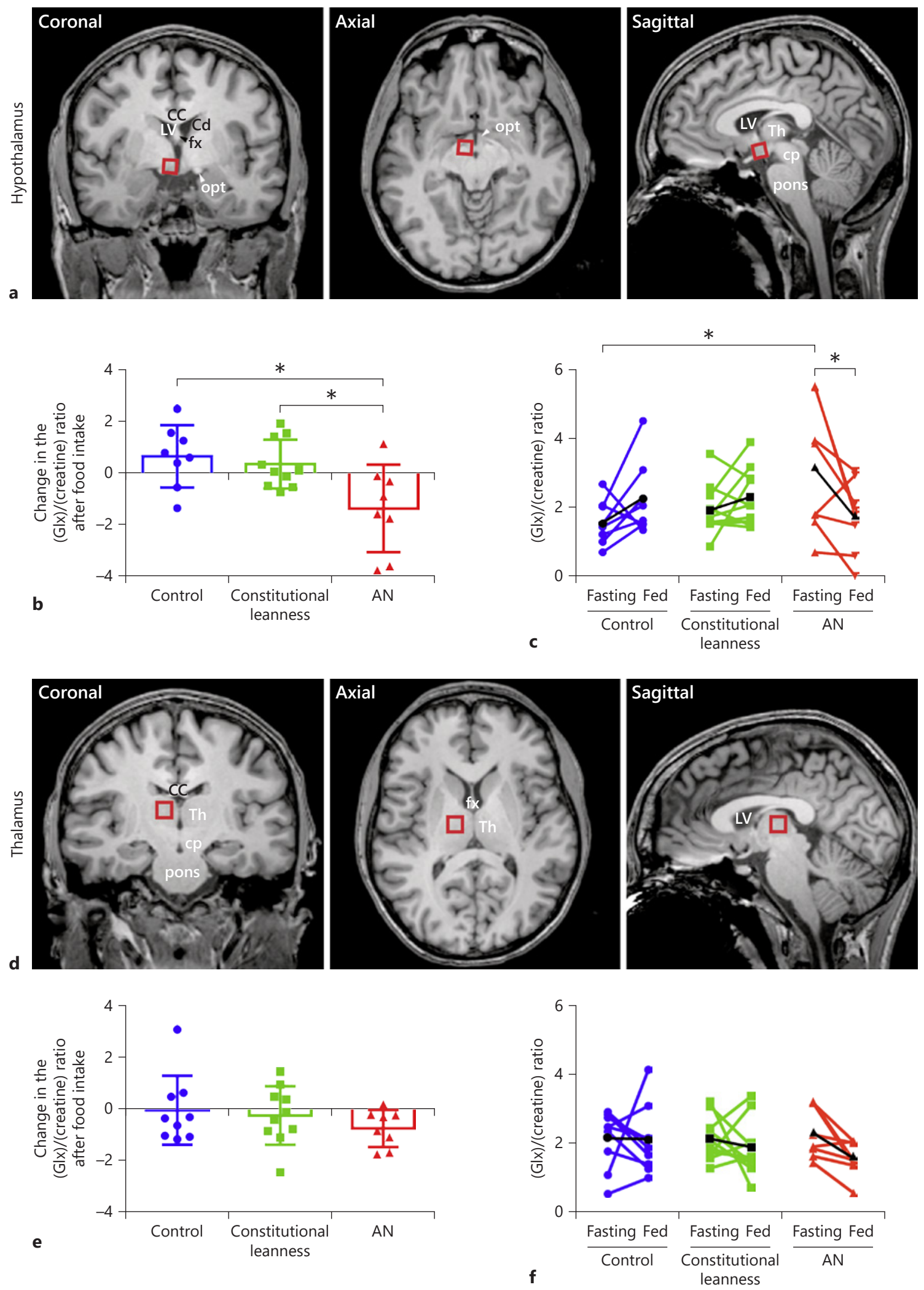

(For legend see next page.) 
after feeding, the ratio of Glx-to-creatine concentrations in the hypothalamus dropped to below prefeeding levels in AN patients (AN fasting vs. AN fed $t_{(23)}=2.964, p=$ $0.021, n=8$ persons per group; Fig. 1c), in contrast to control (control fasting vs. Control fed $t_{(23)}=1.454, p=$ $0.406, n=8$ persons per group) and constitutionally lean participants (Lean fasting vs. Lean fed $t_{(23)}=0.891, p=$ $0.764, n=10$ persons per group), who showed a slight increase (Fig. 1c). The calibrated breakfast thus induced an acute decrease in the ratio of Glx-to-creatine concentrations only in AN patients (Fig. 1c), reducing it to values comparable to those in control participants before breakfast (Fig. 1c). Such a divergence in ratios between AN and normal-weight or lean individuals and the corresponding feeding-induced changes were not seen in the thalamus (Fig. 1e, f, online suppl. Table S3, S4). The ratio of NAA to creatine concentrations did not differ between the 3 groups of women and displayed no significant change in response to feeding in either the hypothalamus (online suppl. Fig. S1a and Table S1) or the thalamus (online suppl. Fig. S1b and Table S3). Altogether our MRS findings suggest a glutamatergic dysfunction in the hypothalamus of patients with $\mathrm{AN}$, that is, an inversion of the glutamatergic response to feeding combined with higher baseline levels in the fasting state. Rodent studies using advanced genetic tools have recently demonstrated that the selective activation of discrete glutamatergic neuronal populations in the ARH and in the LHA mediates satiety in mice fed ad libitum [33] and suppresses feeding in food-deprived mice [34], respectively. Unfortunately, due to physical limitations (3T magnet; $60-\mathrm{cm}$ tunnel diameter; min-acquisition time) and ethical standards (short scanning times in nonanesthetized human volunteers), subregions of the hypothalamus are not amenable to scrutiny using MRS in the brain of living humans.

Glutamate and glutamine have several roles that are not related to neurotransmission. However, since Glx levels were measured both prior to and after a functional

Fig. 1. Hypothalamic glutamine/glutamate levels drop after feeding in individuals with AN. MRS analysis of the hypothalamus $(\mathbf{a}-\mathbf{c})$ and thalamus $(\mathbf{d}-\mathbf{f})$ using the volumes of interest shown in a and $\mathbf{d}$, respectively. Effect of food intake on the ratios of the concentration of Glx to that of creatine $(\mathbf{b}, \mathbf{c}, \mathbf{e}, \mathbf{f})$ in the hypothalamus $(\mathbf{b}, \mathbf{c})$ and the thalamus $(\mathbf{e}, \mathbf{f})$ of participants with a normal body mass index (control), constitutional leanness or AN.* $p<0.05$; the exact $p$ values and statistical analyses are detailed in the text. Cc, corpus callosum; Cd, caudate nucleus; fx, fornix; opt, optic tract, Th, thalamus; cp, cerebral peduncle; LV, lateral ventricle; Glx, glutamate/glutamine.

Hypothalamic Defects in AN task, that is, feeding, the changes we see likely reflect glutamatergic metabolism related to neuronal activation [31, 35]. Because cellular glutamate changes are tightly linked to synaptic plasticity [36], the apparent glutamatergic alterations observed at the macroscopic level are likely to reflect experience-related plasticity as well. In this context, our data raise the intriguing possibility that glutamatergic dysfunction plays a role in the pathophysiology of AN, or indeed, of other eating disorders. Targeting the glutamatergic drive, which normally suppresses feeding $[33,34,37]$, could thus hold therapeutic potential for treating this disorder. Moreover, the similarities between human AN and the constitutive activation of hypothalamic glutamatergic neuronal populations in mice suggest that the latter could constitute a novel and appropriate animal model for further studies of this disease.

In addition to glutamate, the hypothalamus also contains abundant neuronal populations releasing the neurotransmitter GABA, including the orexigenic neurons expressing AgRP and NPY in the ARH $[12,13]$ and cells in the LHA $[13,16]$; selective activation of GABA-producing neurons in the LHA produces voracious feeding $[17,38]$. Glutamatergic and GABAergic neurons in the hypothalamus are thus poised to exert opposing effects on food intake. Hence, it is tempting to speculate that GABA levels may mirror changes in glutamate levels before and after feeding in AN patients. Assessment of GABA levels using MRS requires the use of the MEGAPRESS method that has to be run separately from the classical sequence allowing the direct detection of Glx and other metabolites [39]. The use of this sequence implies to increase the scan time for the volunteers and this is something that could not be done in the present protocol for ethical standards (i.e., our participants have indeed been subjected to 2 consecutive sessions of $45 \mathrm{~min}$, which is the maximum acceptable scan time in unanesthetized and live humans). Further studies will thus need to be designed to investigate the putative changes in GABA levels with feeding in the hypothalamus of AN patients and controls in the future.

\section{The Arcuate Nucleus and LHA Show Inverse Malwiring in $A N$}

To investigate changes in the microstructure of the hypothalamus that could underlie these functional changes, we next manually segmented each hypothalamic structure or nucleus using high-resolution anatomical MRI sequences [10] for all 30 participants individually (Fig. 2; online suppl. Fig. S2). Each of the hypothalamic segments thus constituted a region of interest for diffusion-weight- 


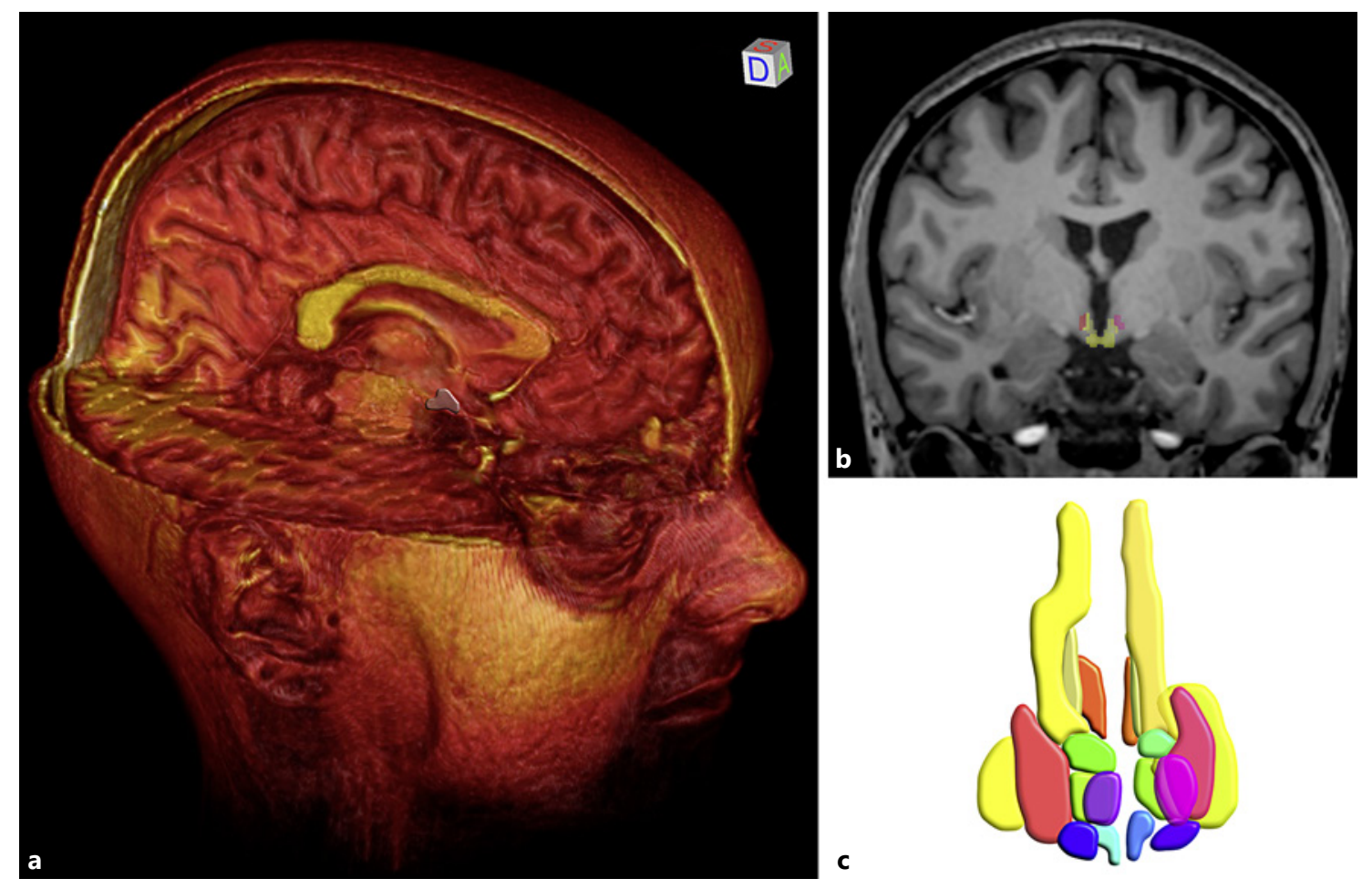

Fig. 2. Anatomically based segmentation of the hypothalamus in a single subject. a Three-dimensional reconstruction of the head of a participant using the Osirix software, with the hypothalamus drawn in gray. $\mathbf{b}$ Coronal section of a high-resolution anatomical image used to identify the distinct regions of the hypothalamus according to previously established anatomical landmarks [10] (see Materials and Methods). Individual hypothalamic nuclei have been assigned color codes as elaborated in online supplementary Figure S2. c Three-dimensional rendering of the segmented hypothalamus.

ed imaging after their transformation into diffusion space. We then used a probabilistic tractography algorithm to determine the number of fibers passing through or present in the distinct ROI in a single subject [9]. The results showed that both AN and constitutional leanness were associated with a profound reduction in the number of fibers in the ARH (one-way ANOVA: $F_{(2,22)}=5.059, P=$ 0.016 ; Tukey's multiple comparison test: Control vs. Lean $q_{(22)}=4.073, p=0.023, n=8$ and 9 persons per group and Control vs. AN $q_{(22)}=3.733, p=0.038, n=8$ persons per group, online suppl. Table S5; Fig. 3a). In contrast, tractography data revealed that the average density of fibers in the LHA was significantly higher only in patients with AN (one-way ANOVA: $F_{(2,27)}=4.345, p=0.023$; Tukey's multiple comparison test: Control vs. AN $q_{(27)}=3.832$, $p=0.03, n=10$ persons per group, online suppl. Table S5) but not in participants with constitutional leanness (Control vs. Lean $q_{(27)}=0.495, p=0.935, n=10$ persons per group; Fig. $3 \mathrm{~b}$ ). Even though diffusion tensor imaging does not enable us to distinguish between incoming and outgoing fibers (or fibers of passage) in a given region of interest, fiber tracts connecting to the ARH were shown to travel via the LHA, the dorsomedial hypothalamic nucleus, the paraventricular hypothalamic nucleus and the paraventricular thalamic nucleus (Fig. 3a), hypothalamic and extrahypothalamic structures to which ARH neurons are known to project in rodents $[12,13,40]$. No differences between groups were noted in the other hypothalamic ROI analyzed (online suppl. Fig. S3 and Table S5). These data provide direct evidence that conditions with a low body mass index may be associated with an alteration of the connectivity of the ARH, a phenomenon that is, intriguingly, reminiscent of observations in mice [41]. Indeed, mouse studies have demonstrated that in animals exhibiting constitutionally low body weight due to nutritional manipulation during postnatal development, projections from the ARH to its hypothalamic targets, which develop relatively late in postnatal life [42-45], are sig- 
Fig. 3. AN increases the connectivity of the LHA while decreasing the wiring of the ARH. DWI showing tractography analysis from 2 ROI transformed into diffusion space (white labels) and corresponding to the ARH (a) and the LHA (b). The graphs show the number of fibers present in the $\mathrm{ARH}(\mathbf{a})$ and the LHA (b). The coronal anatomical image on which tractography data have been superimposed in $\mathbf{a}$ and $\mathbf{b}$ corresponds to anatomical plate 38 of the anatomical atlas of the human brain [27]. ${ }^{*} p<$ 0.05 ; the exact $p$ values and statistical analyses are detailed in the text. DMH, dorsomedial hypothalamus; LV, lateral ventricle; $\mathrm{PaPo}$, paraventricular hypothalamic nucleus; PV, paraventricular nucleus of the thalamus; ARH, arcuate nucleus of the hypothalamus; LHA, lateral hypothalamic area.
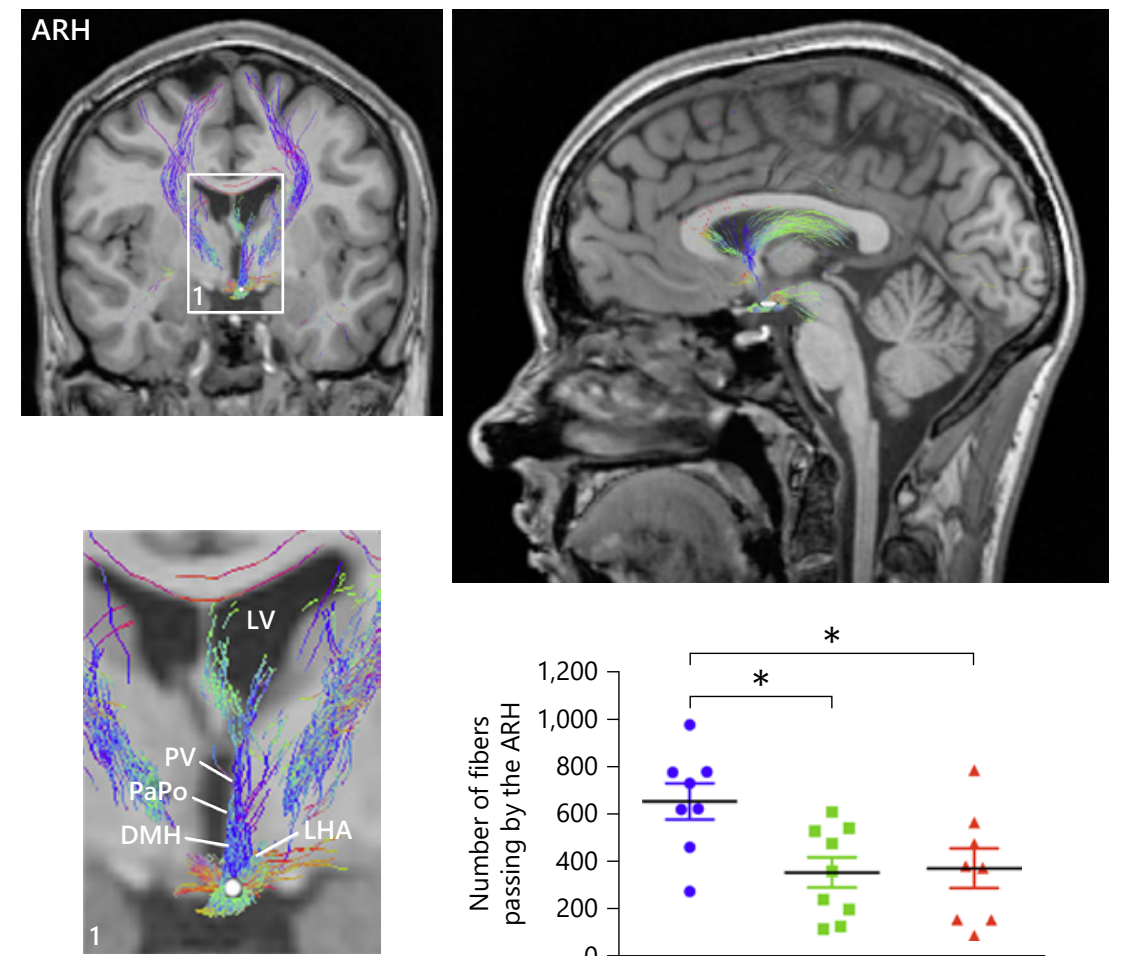

a
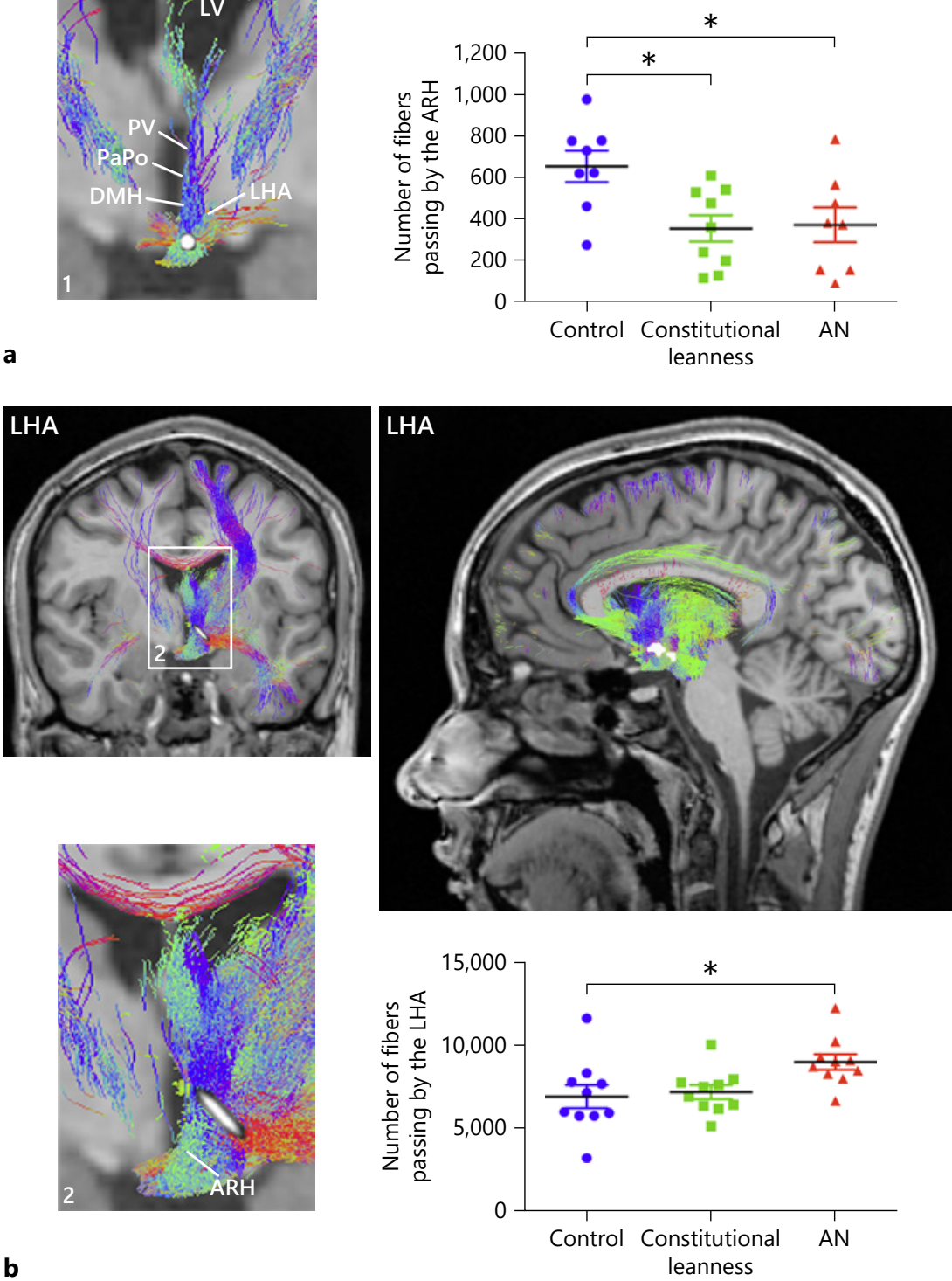

b 
nificantly and permanently decreased when compared to mice with normal body weight [41]. An alternative hypothesis is that axons projecting to or from the ARH undergo selective degeneration, as has been shown in a genetic animal model of anorexia [46] via a process involving inflammation [47] and mitochondrial dysfunction [48]. However, our spectroscopic data showing that hypothalamic levels of NAA, routinely used in the clinic to detect neurodegenerative processes [49], are unchanged compared to controls (online suppl. Fig. S1a) argues against the involvement of a degenerative process in the hypothalamus of individuals with a low body mass index. Interestingly, when the lean phenotype is associated with maladaptive feeding behavior, that is, in participants with $\mathrm{AN}$, the decrease in the ARH fiber density, also evident in constitutionally lean individuals, is accompanied by a more specific increase in connectivity of the LHA (Fig. 3), a critical neuroanatomical substrate for motivated behavior $[13,16]$. In line with this putative alteration of neuronal wiring, genome-wide association studies in AN have identified genetic variants in neurodevelopmental genes regulating synapse and neuronal network formation [50].

This study compared metabolites in the hypothalamus under fasting conditions and after the ingestion of a meal, and the structure of the underlying neuroanatomical substrate in individuals with $\mathrm{AN}$ and constitutional leanness. Prior functional MRI studies, limited to extra-hypothalamic structures, have examined the effect of feeding and of the peripheral administration of metabolically active molecules on appetite and brain activation in healthy volunteers [51,52], and of the neural responses to the passive viewing of food [53-55] or food choice in AN [56]. Our results raise the provocative idea that the origin of the disconnect between the body's needs and behavioral choices in patients with AN could lie in the hypothalamus, the metabolic window to the brain, which could fail to accurately sense circulating metabolic signals $[57,58]$ and post-ingestive cues $[59,60]$, and thus relay erroneous information to higher decision centers. The recent correlation of genetic variants, including some expressed in the hypothalamus, with metabolic traits in $\mathrm{AN}[5,6]$, provides further corroboration for this hypothesis. In addition, our data suggest that, as proposed for obesity [61], AN could be a neurodevelopmental disorder in which an individual's predisposition is determined by the malwiring and dysfunction of hypothalamic nuclei that communicate with the neuroendocrine and autonomous nervous systems and key decision centers in the telencephalon via subcortical circuits. These centers are crucial for certain important and interrelated functions such as the control

of energy homeostasis and food intake, the attribution of reward value to food, and bodily representation. Our results shed new light on the pathophysiology of AN by providing potential neurobiological explanations to the aberrant response to low energy state and may thus prove key to developing effective treatments for this debilitating illness.

\section{Statement of Ethics}

The study was carried out with the approval of the Comite de Protection des Personnes Nord Ouest IV (No. EudraCT: 2012A01331-42), and informed consent was obtained from all included subjects.

\section{Disclosure Statement}

The authors declare no conflict of interest.

\section{Funding Sources}

This research was supported by the Agence Nationale de la Recherche (ANR-16-CE37-0006, France to V.P.), a NARSAD YI Grant from the Brain and Behavior Research Foundation (to I.A.K.N), the Swedish Foundations; Brain and Lundbeck (to I.A.K.N) and the NEUROBESE International Associated Laboratory (Inserm, University of Lille, Children Hospital of Los Angeles, to S.G.B. and V.P.), and the National Institutes of Health (Grants DK84142, DK102780, and DK118401 to S.G.B.).

\section{Author Contribution}

V.F., M.B., I.A.K.N., and V.P.: designed the experiments. V.F., J.V., and M.P.: screened and included the volunteers in the study. V.F., P.J.-T., R.L., M.V., M.M., D.L., A.L., M.T.S., P.C., A.S., and V.P.: performed the experiments. V.F., M.B., P.J.-T., R.L., M.V., and V.P.: analyzed the data. V.F. and S.V.: performed the statistical analyses. All authors discussed the results and made edits to the manuscript. V.F., M.B., I.A.K.N., and V.P.: wrote the manuscript.

References
1 Treasure J, Zipfel S, Micali N, Wade T, Stice E, Claudino A, et al. Anorexia nervosa. Nat Rev Dis Primers. 2015 Nov;1(1):15074.

2 Zipfel S, Giel KE, Bulik CM, Hay P, Schmidt U. Anorexia nervosa: aetiology, assessment, and treatment. Lancet Psychiatry. 2015 Dec; 2(12):1099-111.

3 Kaye WH, Wierenga CE, Bailer UF, Simmons AN, Bischoff-Grethe A. Nothing tastes as good as skinny feels: the neurobiology of anorexia nervosa. Trends Neurosci. 2013 Feb; 36(2):110-20. 
4 Schorr M, Miller KK. The endocrine manifestations of anorexia nervosa: mechanisms and management. Nat Rev Endocrinol. 2017 Mar; 13(3):174-86

5 Duncan L, Yilmaz Z, Gaspar H, Walters R, Goldstein J, Anttila V, et al.; Eating Disorders Working Group of the Psychiatric Genomics Consortium. Significant Locus and Metabolic Genetic Correlations Revealed in GenomeWide Association Study of Anorexia Nervosa. Am J Psychiatry. 2017 Sep;174(9):850-8.

6 Hinney A, Kesselmeier M, Jall S, Volckmar AL, Föcker $M$, Antel $J$, et al.; GCAN, WTCCC3; GIANT, SFA Grant, EGG; Price Foundation Collaborative Group, Children's Hospital of Philadelphia/Price Foundation. Evidence for three genetic loci involved in both anorexia nervosa risk and variation of body mass index. Mol Psychiatry. 2017 Feb; 22(2):321-2.

7 King JA, Frank GK, Thompson PM, Ehrlich S. Structural Neuroimaging of Anorexia Nervosa: Future Directions in the Quest for Mechanisms Underlying Dynamic Alterations. Biol Psychiatry. 2018 Feb;83(3):224-34.

8 Baroncini M, Jissendi P, Catteau-Jonard S, Dewailly D, Pruvo JP, Francke JP, et al. Sex steroid hormones-related structural plasticity in the human hypothalamus. Neuroimage. 2010 Apr;50(2):428-33.

9 Behrens TE, Johansen-Berg H, Woolrich MW, Smith SM, Wheeler-Kingshott CA, Boulby PA, et al. Non-invasive mapping of connections between human thalamus and cortex using diffusion imaging. Nat Neurosci. 2003 Jul;6(7):750-7.

10 Baroncini M, Jissendi P, Balland E, Besson P, Pruvo JP, Francke JP, et al. MRI atlas of the human hypothalamus. Neuroimage. 2012 Jan;59(1):168-80.

11 Gautron L, Elmquist JK, Williams KW. Neural control of energy balance: translating circuits to therapies. Cell. 2015 Mar;161(1):13345.

12 Andermann ML, Lowell BB. Toward a Wiring Diagram Understanding of Appetite Control. Neuron. 2017 Aug;95(4):757-78.

13 Sternson SM, Eiselt AK. Three Pillars for the Neural Control of Appetite. Annu Rev Physiol. 2017 Feb;79(1):401-23.

14 Zimmerman CA, Leib DE, Knight ZA. Neural circuits underlying thirst and fluid homeostasis. Nat Rev Neurosci. 2017 Aug;18(8):45969.

15 Livneh Y, Ramesh RN, Burgess CR, Levandowski KM, Madara JC, Fenselau H, et al. Homeostatic circuits selectively gate food cue responses in insular cortex. Nature. 2017 Jun; 546(7660):611-6.

16 Stuber GD, Wise RA. Lateral hypothalamic circuits for feeding and reward. Nat Neurosci. 2016 Feb;19(2):198-205.

17 Carus-Cadavieco M, Gorbati M, Ye L, Bender F, van der Veldt S, Kosse C, et al. Gamma oscillations organize top-down signalling to hypothalamus and enable food seeking. Nature. 2017 Feb;542(7640):232-6.
18 Croizier S, Chometton S, Fellmann D, Risold PY. Characterization of a mammalian prosencephalic functional plan. Front Neuroanat. 2015 Jan;8:161.

19 Mishell DR Jr, Thorneycroft IH, Nakamura RM, Nagata Y, Stone SC. Serum estradiol in women ingesting combination oral contraceptive steroids. Am J Obstet Gynecol. 1972 Dec;114(7):923-8.

20 Estour B, Germain N, Diconne E, Frere D, Cottet-Emard JM, Carrot G, et al. Hormonal profile heterogeneity and short-term physical risk in restrictive anorexia nervosa. J Clin Endocrinol Metab. 2010 May;95(5):2203-10.

21 Marshall JC, Kelch RP. Low dose pulsatile gonadotropin-releasing hormone in anorexia nervosa: a model of human pubertal development. J Clin Endocrinol Metab. 1979 Nov; 49(5):712-8.

22 Hemrika DJ, Slaats EH, Kennedy JC, de Vries Robles-Korsen TJ, Schoemaker J. Pulsatile luteinizing hormone patterns in long term oral contraceptive users. J Clin Endocrinol Metab. 1993 Aug;77(2):420-6.

23 de Lauzon B, Romon M, Deschamps V, Lafay L, Borys JM, Karlsson J, et al.; Fleurbaix Laventie Ville Sante Study Group. The ThreeFactor Eating Questionnaire-R18 is able to distinguish among different eating patterns in a general population. J Nutr. 2004 Sep;134(9): 2372-80.

24 Frankenfield DC, Muth ER, Rowe WA. The Harris-Benedict studies of human basal metabolism: history and limitations. J Am Diet Assoc. 1998 Apr;98(4):439-45.

25 Joers JM, Deelchand DK, Kumar A, Moheet A, Seaquist E, Henry PG, et al. Measurement of Hypothalamic Glucose Under Euglycemia and Hyperglycemia by MRI at 3T. J Magn Reson Imaging. 2017 Mar;45(3):681-91.

26 Lemaire JJ, Frew AJ, McArthur D, Gorgulho AA, Alger JR, Salomon N, et al. White matter connectivity of human hypothalamus. Brain Res. 2011 Jan;1371:43-64.

27 Mai JK, Majtanik M, Paxinos G. Atlas of the human brain, ed 4th edition. Academic press, 2016.

28 Provencher SW. Estimation of metabolite concentrations from localized in vivo proton NMR spectra. Magn Reson Med. 1993 Dec; 30(6):672-9.

29 Rohlfing T, Maurer CR Jr. Nonrigid image registration in shared-memory multiprocessor environments with application to brains, breasts, and bees. IEEE Trans Inf Technol Biomed. 2003 Mar;7(1):16-25.

30 Tournier JD, Calamante F, Connelly A. Robust determination of the fibre orientation distribution in diffusion MRI: non-negativity constrained super-resolved spherical deconvolution. Neuroimage. 2007 May;35(4): 1459-72.

31 Stanley JA, Raz N. Functional Magnetic Resonance Spectroscopy: The "New" MRS for Cognitive Neuroscience and Psychiatry Research. Front Psychiatry. 2018 Mar;9:76.
32 Estour B, Marouani N, Sigaud T, Lang F, Fakra E, Ling Y, et al. Differentiating constitutional thinness from anorexia nervosa in DSM 5 era. Psychoneuroendocrinology. 2017 Oct;84:94-100

33 Fenselau H, Campbell JN, Verstegen AM, Madara JC, Xu J, Shah BP, et al. A rapidly acting glutamatergic $\mathrm{ARC} \rightarrow \mathrm{PVH}$ satiety circuit postsynaptically regulated by $\alpha-\mathrm{MSH}$. Nat Neurosci. 2017 Jan;20(1):42-51.

34 Jennings JH, Rizzi G, Stamatakis AM, Ung RL, Stuber GD. The inhibitory circuit architecture of the lateral hypothalamus orchestrates feeding. Science. 2013 Sep;341(6153): $1517-21$.

35 Duarte JMN, Xin L. Magnetic Resonance Spectroscopy in Schizophrenia: Evidence for Glutamatergic Dysfunction and Impaired Energy Metabolism. Neurochem Res. 2019 Jan;44(1):102-16.

36 McEwen BS, Morrison JH. The brain on stress: vulnerability and plasticity of the prefrontal cortex over the life course. Neuron. 2013 Jul;79(1):16-29.

37 Nectow AR, Schneeberger M, Zhang H, Field BC, Renier N, Azevedo E, et al. Identification of a Brainstem Circuit Controlling Feeding. Cell. 2017 Jul;170(3):429-42.e11.

38 Jennings JH, Ung RL, Resendez SL, Stamatakis AM, Taylor JG, Huang J, et al. Visualizing hypothalamic network dynamics for appetitive and consummatory behaviors. Cell. 2015 Jan;160(3):516-27.

39 Puts NA, Edden RA. In vivo magnetic resonance spectroscopy of GABA: a methodological review. Prog Nucl Magn Reson Spectrosc. 2012 Jan;60:29-41.

40 Elmquist JK, Coppari R, Balthasar N, Ichinose $\mathrm{M}$, Lowell BB. Identifying hypothalamic pathways controlling food intake, body weight, and glucose homeostasis. J Comp Neurol. 2005 Dec;493(1):63-71.

41 Caron E, Ciofi P, Prevot V, Bouret SG. Alteration in neonatal nutrition causes perturbations in hypothalamic neural circuits controlling reproductive function. J Neurosci. 2012 Aug;32(33):11486-94.

42 Bouret SG, Draper SJ, Simerly RB. Trophic action of leptin on hypothalamic neurons that regulatefeeding. Science. 2004 Apr;304(5667): 108-10.

43 Bouret SG, Draper SJ, Simerly RB. Formation of projection pathways from the arcuate nucleus of the hypothalamus to hypothalamic regions implicated in the neural control of feeding behavior in mice. J Neurosci. 2004 Mar;24(11):2797-805.

44 Vogt MC, Paeger L, Hess S, Steculorum SM, Awazawa M, Hampel B, et al. Neonatal insulin action impairs hypothalamic neurocircuit formation in response to maternal high-fat feeding. Cell. 2014 Jan;156(3):495-509.

45 Steculorum SM, Collden G, Coupe B, Croizier S, Lockie S, Andrews ZB, et al. Neonatal ghrelin programs development of hypothalamic feeding circuits. J Clin Invest. 2015 Feb; 125(2):846-58 
46 Broberger C, Johansen J, Johansson C, Schalling M, Hökfelt T. The neuropeptide Y/agouti gene-related protein (AGRP) brain circuitry in normal, anorectic, and monosodium glutamate-treated mice. Proc Natl Acad Sci USA. 1998 Dec;95(25):15043-8.

47 Nilsson IA, Thams S, Lindfors C, Bergstrand A, Cullheim S, Hökfelt T, et al. Evidence of hypothalamic degeneration in the anorectic anx/anx mouse. Glia. 2011 Jan;59(1):45-57.

48 Lindfors C, Nilsson IA, Garcia-Roves PM, Zuberi AR, Karimi M, Donahue LR, et al. Hypothalamic mitochondrial dysfunction associated with anorexia in the anx/anx mouse. Proc Natl Acad Sci USA. 2011 Nov; 108(44): 18108-13.

49 Kantarci K. Magnetic resonance spectroscopy in common dementias. Neuroimaging Clin $\mathrm{N}$ Am. 2013 Aug;23(3):393-406.

50 Boraska V, Franklin CS, Floyd JA, Thornton LM, Huckins LM, Southam L, et al.; Wellcome Trust Case Control Consortium 3. A genome-wide association study of anorexia nervosa. Mol Psychiatry. 2014 Oct;19(10): 1085-94.
51 De Silva A, Salem V, Long CJ, Makwana A, Newbould RD, Rabiner EA, et al. The gut hormones PYY 3-36 and GLP-1 7-36 amide reduce food intake and modulate brain activity in appetite centers in humans. Cell Metab. 2011 Nov; 14(5):700-6.

52 Luo S, Monterosso JR, Sarpelleh K, Page KA. Differential effects of fructose versus glucose on brain and appetitive responses to food cues and decisions for food rewards. Proc Natl Acad Sci USA. 2015 May;112(20):6509-14.

53 Uher R, Brammer MJ, Murphy T, Campbell IC, Ng VW, Williams SC, et al. Recovery and chronicity in anorexia nervosa: brain activity associated with differential outcomes. Biol Psychiatry. 2003 Nov;54(9):934-42.

54 Ellison Z, Foong J, Howard R, Bullmore E, Williams S, Treasure J. Functional anatomy of calorie fear in anorexia nervosa. Lancet. 1998 Oct;352(9135):1192.

55 Kühn AB, Feis DL, Schilbach L, Kracht L, Hess ME, Mauer J, et al. FTO gene variant modulates the neural correlates of visual food perception. Neuroimage. 2016 Mar;128:2131.

56 Foerde K, Steinglass JE, Shohamy D, Walsh BT. Neural mechanisms supporting maladaptive food choices in anorexia nervosa. Nat Neurosci. 2015 Nov;18(11):1571-3.
57 Galusca B, Jeandel L, Germain N, Alexandre $\mathrm{D}$, Leprince J, Anouar Y, et al. Orexigenic neuropeptide 26RFa: new evidence for an adaptive profile of appetite regulation in anorexia nervosa. J Clin Endocrinol Metab. 2012 Jun;97(6):2012-8.

58 Prevot V, Dehouck B, Sharif A, Ciofi P, Giacobini $\mathrm{P}$, Clasadonte J. The versatile tanycyte: A hypothalamic integrator of reproduction and energy metabolism. Endocr Rev. 2018 Jun;39(3):333-68.

$59 \mathrm{Su}$ Z, Alhadeff AL, Betley JN. Nutritive, Postingestive Signals Are the Primary Regulators of AgRP Neuron Activity. Cell Rep. 2017 Dec; 21(10):2724-36.

60 Clemmensen C, Müller TD, Woods SC, Berthoud HR, Seeley RJ, Tschöp MH. Gut-Brain Cross-Talk in Metabolic Control. Cell. 2017 Feb;168(5):758-74.

61 Bouret SG. Development of Hypothalamic Circuits That Control Food Intake and Energy Balance. In; Harris RB, editor. Appetite and Food Intake: Central Control. Boca Raton; 2017. 